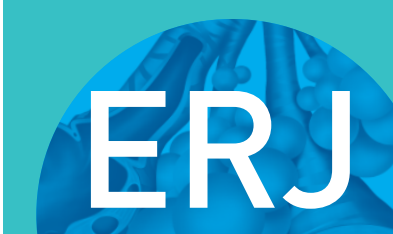

open research
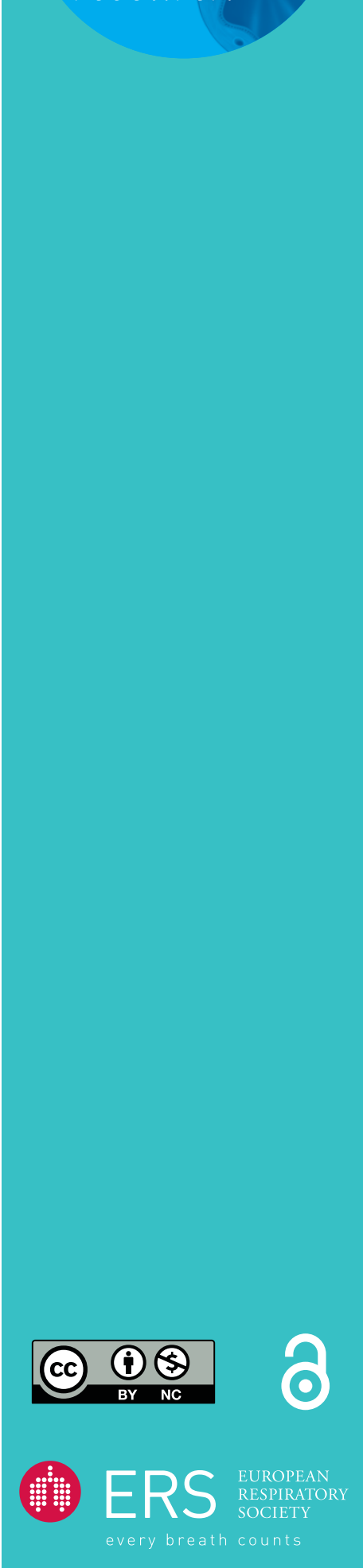

\section{Regional lung function in nonsmokers and asymptomatic current and former smokers}

\author{
Barbara Vogt ${ }^{1}$, Kathinka Deuß ${ }^{1}$, Victoria Hennig ${ }^{1}$, Zhanqi Zhao ${ }^{2,3}$, \\ Ingmar Lautenschläger ${ }^{1}$, Norbert Weiler ${ }^{1}$ and Inéz Frerichs ${ }^{1}$
}

Affiliations: ${ }^{1}$ Dept of Anesthesiology and Intensive Care Medicine, University Medical Center SchleswigHolstein, Campus Kiel, Kiel, Germany. ${ }^{2}$ Dept of Biomedical Engineering, Fourth Military Medical University, Xi'an, China. ${ }^{3}$ Dept of Biomedical Engineering, Furtwangen University, Villingen-Schwenningen, Germany.

Correspondence: Barbara Vogt, Dept of Anesthesiology and Intensive Care Medicine, University Medical Center Schleswig-Holstein, Campus Kiel, Arnold-Heller-Str. 3, Haus 12, D-24105 Kiel, Germany. E-mail: barbara.vogt@uksh.de

ABSTRACT Electrical impedance tomography (EIT) is able to detect rapid lung volume changes during breathing. The aim of our observational study was to characterise the heterogeneity of regional ventilation distribution in lung-healthy adults by EIT and to detect the possible impact of tobacco consumption.

A total of 219 nonsmokers, asymptomatic ex-smokers and current smokers were examined during forced full expiration using EIT. Forced expiratory volume in $1 \mathrm{~s}$ (FEV1), forced vital capacity (FVC) and FEV $1 / F V C$ were determined in 836 EIT image pixels for the analysis of spatial and temporal ventilation distribution. Coefficients of variation $(\mathrm{CVs})$ of these pixel values were calculated. Histograms and medians of FEV1/FVCEIT and times required to exhale 50\%, 75\%, 90\% of FVCEIT (t50, t75 and t90) were generated.

$\mathrm{CV}$ of $\mathrm{FEV} / \mathrm{FVCEIT}$ distinguished among all groups (mean \pm SD: nonsmokers $0.43 \pm 0.05$, ex-smokers $0.52 \pm 0.09$, smokers $0.62 \pm 0.16$ ). Histograms of FEV1/FVCEIT differentiated between nonsmokers and the other groups $(\mathrm{p}<0.0001)$. Medians of $\mathrm{t} 50, \mathrm{t} 75$ and $\mathrm{t} 90$ showed the lowest values in nonsmokers. Median $\mathrm{t} 90$ separated all groups (median (interquartile range): nonsmokers 0.82 (0.67-1.15), ex-smokers 1.41 (1.03-2.21), smokers $1.91(1.33-3.53))$.

EIT detects regional ventilation heterogeneity during forced expiration in healthy nonsmokers and its increase in asymptomatic former and current smokers. Therefore, EIT-derived reference values should only be collected from nonsmoking lung-healthy adults.

@ERSpublications

EIT assesses the spatial and temporal distribution of lung function in adults without pulmonary disease, and detects its increased heterogeneity in former and current smokers compared with nonsmokers. EIT reference values should originate from nonsmokers. http://bit.ly/2wsJQWD

Cite this article as: Vogt B, Deuß K, Hennig V, et al. Regional lung function in nonsmokers and asymptomatic current and former smokers. ERJ Open Res 2019; 5: 00240-2018 [https://doi.org/ 10.1183/23120541.00240-2018].

Received: Dec 102018 | Accepted after revision: May 012019

Copyright $\odot E R S$ 2019. This article is open access and distributed under the terms of the Creative Commons Attribution Non-Commercial Licence 4.0. 


\section{Introduction}

Pulmonary disorders are associated with heterogeneity of ventilation distribution [1-4]. Pulmonary function testing (PFT) and imaging are frequently used for the assessment of lung impairment, most commonly spirometry, chest radiography and computed tomography (CT). Spirometry determines the global lung function derived from measurements at the airway opening. It is not able to assess the regional lung function. Radiography serves as an initial imaging examination for the rough detection of lung changes. CT provides the anatomical information on the lung structure and is able to present structural changes like atelectasis or bronchiectasis; however, both imaging modalities are associated with radiation exposure.

Electrical impedance tomography (EIT) is a functional imaging modality capable of detecting rapid air content changes as required by PFT [5]. Its use is new in pneumology [6, 7]. EIT assesses the regional lung function and is able to identify ventilation heterogeneities directly in the examined region of the chest in spontaneously breathing subjects $[8,9]$. Because of its noninvasiveness, radiation-free measuring principle and easy bedside use, EIT can be applied at any age [10-15]. In order to correctly interpret the impairment of regional lung function and its development over time in patients with lung diseases, reference values of regional lung function parameters derived from a lung-healthy population are needed. No systematic large EIT data exist on regional lung function in lung-healthy adults. Therefore, we examined a population of asymptomatic lung-healthy adults using EIT. In order to verify whether subtle functional changes in ventilation distribution are detectable, we included three groups of subjects in our study population: nonsmokers and asymptomatic former and current smokers.

The goal of the study was the characterisation of the ventilation distribution in a lung-healthy, asymptomatic population and the determination of tobacco's influence on a regional level using EIT. The obtained results are intended to serve as reference values for patients with lung diseases.

\section{Material and methods}

The observational monocentric study was approved by the institutional ethics committee and informed written consent was obtained from each studied subject. Between August 2013 and October 2013, PFT using EIT was carried out in 219 lung-healthy subjects during a standardised forced vital capacity (FVC) manoeuvre [16] during an average of $90 \mathrm{~s}$. A total of 36 subjects were excluded from further data analysis due to their inability to adequately perform the manoeuvre. From the remaining 183 subjects, a further 5 were excluded because of inadequate electrode contact, necessary for proper EIT scanning. The remaining 178 lung-healthy asymptomatic subjects consisted of 64 nonsmokers, 68 past smokers and 46 current smokers (figure 1). The examined patients also served as a study population in a separate examination regarding the preoperative pulmonary risk assessment using the new spirometric reference values of the

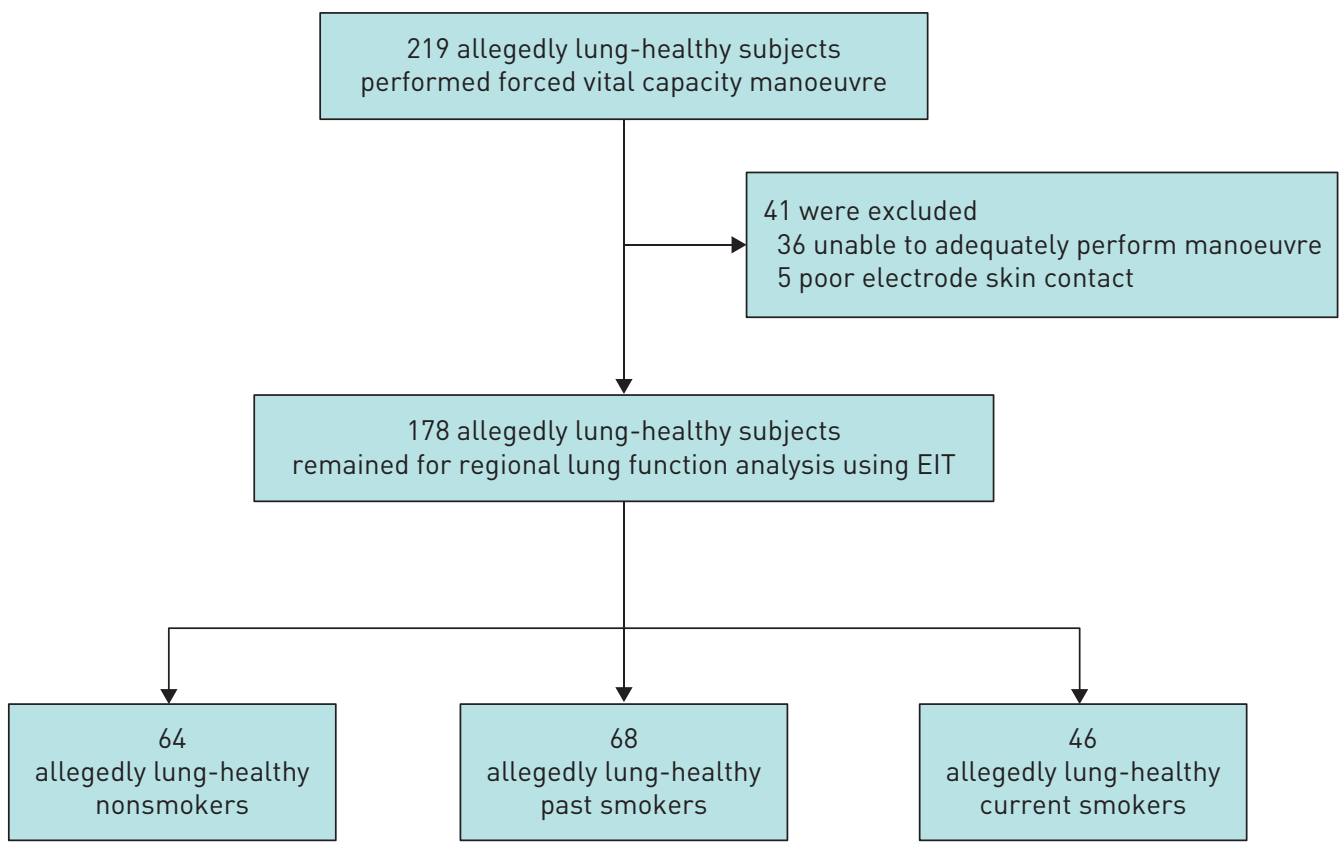

FIGURE 1 Enrolment and follow-up of the study participants. EIT: electrical impedance tomography. 
worldwide Global Lung Initiative [17]. Nonsmokers were defined as subjects who did not smoke more than 50 cigarettes in their whole life, past smokers as subjects that managed to quit smoking for more than 6 months before the study and current smokers as subjects who were smoking up to the inclusion in the study. All subjects had no history of chronic obstructive pulmonary disease or other pulmonary diseases or any pulmonary symptoms. They did not use any pulmonary effective drugs. Not included in the study were subjects younger than 18 years and subjects having a contraindication to an FVC manoeuvre, such as cerebral aneurysm.

A total of 16 electrodes (BlueSensor BR-50-K, Ambu, Ballerup, Denmark) were attached on the chest circumference at the 4-5th intercostal space for the EIT data collection and one reference electrode on the abdomen of each subject. All participants were studied in the sitting position using the Goe-MF II device

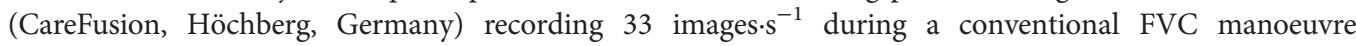
consisting of full inspiration from residual volume to total lung capacity (TLC) with subsequent forced full expiration.

The EIT measuring principle is based on repetitive alternating current applications $\left(5 \mathrm{~mA}_{\mathrm{rms}}\right.$ and $\left.50 \mathrm{kHz}\right)$ between 16 adjacent pairs of electrodes. During each current application over one adjacent electrode pair, 13 remaining passive adjacent electrode pairs measured the resulting voltages. A sequence of 2000 to 3300 raw EIT images (each consisting of 836 image pixels) was acquired in each study subject during the examination. The images were generated by the reconstruction algorithm GREIT [18]. They showed the differences between the instantaneous $(Z)$ and reference pixel impedance $\left(Z_{\text {ref }}\right)$ normalised by $Z_{\text {ref }}$ $\left(\left(Z-Z_{\text {ref }}\right) / Z_{\text {ref }}\right)$. Pixel $Z_{\text {ref }}$ corresponded to the average pixel $Z$ during the stable tidal breathing period at the beginning of the measurement before the forced manoeuvre. These values are given as "relative impedance change" (rel. $\Delta \mathrm{Z}$ ). The pixel EIT waveforms of rel. $\Delta \mathrm{Z}$ in each of the image pixels were used for further analysis of regional lung volumes and expiratory times. For the detailed analysis of regional lung function, we identified the beginning and the end of the forced full expiration in every EIT waveform. Thereafter we calculated the following values in each EIT pixel: forced expiratory volume in $1 \mathrm{~s}$ (FEV1,EIT) as the difference between rel. $\Delta \mathrm{Z}$ at TLC and after $1 \mathrm{~s}$ of forced expiration, FVC (FVCEIT) as the difference between rel. $\Delta Z$ at TLC and the end of forced expiration and the FEV1/FVCEIT ratio. In order to characterise the heterogeneity of ventilation distribution, we calculated the coefficient of variation $(\mathrm{CV})$ from the 836 values of each EIT-derived lung function parameter. Furthermore, we generated histograms of the pixel values of FEV1/FVCEIT, presenting the spatial ventilation distribution in more detail. The temporal ventilation distribution was assessed by histograms of pixel expiratory times required to exhale $50 \%(\mathrm{t} 50), 75 \%(\mathrm{t} 75)$ and $90 \%(\mathrm{t} 90)$ of FVC.

Statistical analysis was carried out by GraphPad Prism version 5.01 (GraphPad Software, San Diego, CA, USA). The differences in all EIT-derived regional lung function parameters among the three studied groups were determined by one-way ANOVA with Bonferroni's multiple comparison post-test. A p-value $<0.017$ was considered significant.

\section{Results}

We analysed 64 nonsmokers, 68 past smokers and 46 currents smokers. The studied former smokers were older than current and nonsmokers and they showed lower smoking intensity (indicated by pack-years) than current smokers. The proportion of women compared with men was higher in nonsmokers than in the other two groups.

The physical characteristics of the study participants and the corresponding spirometric lung function values are given in table 1 .

Subtle ventilation differences like reduced ventilation impedance signal variation or regional deficit in ventilation were already discernible in functional EIT images before the quantitative analysis of ventilation heterogeneity. Example images are given in figure 2 presenting functional EIT images obtained in three subjects during FVC: one nonsmoker, one former smoker and one current smoker.

The CV value of regional FEV1/FVCEIT was the highest in smokers followed by former smokers and nonsmokers. It distinguished among the three groups $(\mathrm{p}<0.0001)$ and indicated the most homogenous ventilation distribution in nonsmokers and the highest degree of ventilation heterogeneity in allegedly lung-healthy smokers. The CV of FEV1,EIT and FVCEIT did not show any significant differences among the three groups (figure 3).

The frequency distributions of all pixel values of FEV1/FVCEIT presented as histograms highlighted the spatial ventilation heterogeneity (figure 4). In lung-healthy nonsmokers the values were higher with a rather narrow peak at higher values (figure 4a), whereas the former and current smokers showed flat distributions (figure $4 \mathrm{~b}$ and $\mathrm{c}$ ); in smokers the values were shifted towards left to lower values (figure $4 \mathrm{c}$ ). 


\begin{tabular}{|c|c|c|c|c|}
\hline & Nonsmokers & Former smokers & Current smokers & p-value \\
\hline Males/females & $29 / 35$ & $40 / 28$ & $22 / 24$ & \\
\hline Age years & $45(18-80)$ & $59(23-83)^{\#}$ & 47 (19-73) & 0.0091 \\
\hline Height $\mathrm{cm}$ & $172(156-194)$ & $174(139-194)$ & $176(160-205)$ & 0.6208 \\
\hline Weight kg & $75(51-125)$ & $80(50-135)$ & $74(50-120)$ & 0.3674 \\
\hline Smoking intensity pack-years & & $15.0(0.2-96)^{\pi}$ & $23(0.3-82)$ & 0.0258 \\
\hline \multicolumn{5}{|l|}{ Z-scores } \\
\hline FEV 1 & $0.22(-2.08-3.35)$ & $-0.07(-3.37-3.32)$ & $-0.92(-4.3-2.02)^{+}$ & $<0.0001$ \\
\hline FVC & $-0.10(-2.8-2.69)$ & $-0.05(-3.49-3.05)$ & $-0.45(-3.19-2.26)$ & 0.0449 \\
\hline $\mathrm{FEV}_{1} / \mathrm{FVC}$ & $0.34(-2.06-3.42)$ & $-0.06(-2.56-1.84)$ & $-0.64(-4.08-1.71)^{\S}$ & $<0.0001$ \\
\hline \multicolumn{5}{|c|}{$\begin{array}{l}\text { Data are presented as median (range) unless otherwise stated. FEV } 1 \text { : forced expiratory volume in } 1 \mathrm{~s} ; \mathrm{FVC} \text { : } \\
\text { forced vital capacity. \#: significant difference between former smokers and nonsmokers and current } \\
\text { smokers; ๆ: significant differences between former and current smokers; }{ }^{+}: \text {significant differences } \\
\text { between current smokers and former smokers and nonsmokers; }{ }^{\S} \text { : significant differences among current } \\
\text { and former smokers and nonsmokers. }\end{array}$} \\
\hline
\end{tabular}

Median of FEV1/FVCEIT distinguished between the lung-healthy nonsmokers (median: 0.97) and the other two groups (median past smokers: 0.87; median smokers: 0.84) $(\mathrm{p}<0.0001)$.

The temporal heterogeneity of ventilation distribution was shown by the frequency distributions of pixel $\mathrm{t} 50, \mathrm{t} 75$ and $\mathrm{t} 90$ presented as histograms (figure 5). Already in t50, there were significant differences between the median value of nonsmokers and the values of past and currents smokers $(p=0.0003)$. The histograms of nonsmokers were characterised by the highest peak and a narrow base when compared with the other two study groups (figure $5 \mathrm{a}, \mathrm{d}$ and $\mathrm{g}$ ). The median of $\mathrm{t} 75$ also discriminated between nonsmokers and the subjects affected by smoking $(\mathrm{p}<0.0001)$. Past and current smokers showed a pixel shift towards higher values (to the right) with a more heterogeneous pixel values distribution pattern than the lung-healthy nonsmokers (figure 5b, e and $\mathrm{h}$ ). The best discrimination among the groups offered the regional t90 values (figure $5 \mathrm{c}, \mathrm{f}$ and $\mathrm{i}$ ). The median values of $\mathrm{t} 90$ were the longest in smokers and the shortest in nonsmokers and this effect was highly significant $(\mathrm{p}<0.0001)$. The distribution of the pixel values of t90 reached the highest degree of ventilation heterogeneity when compared with $\mathrm{t} 50$ and $\mathrm{t} 75$.

The CV values of regional t50, t75 und t90 were able to equally differentiate between the heterogeneities of ventilation distribution in nonsmokers and both past and current smokers $(p<0.0001)$. We did not find any differences between past and current smokers when the $\mathrm{CV}$ of regional expiration times was considered (figure 6).

The corresponding values of the above-mentioned regional parameters of ventilation heterogeneity derived by EIT are given in table 2.

\section{Discussion}

The results of our study showed that EIT-derived regional lung function parameters were able to assess the spatial and temporal ventilation distribution in lung-healthy subjects during a forced expiration

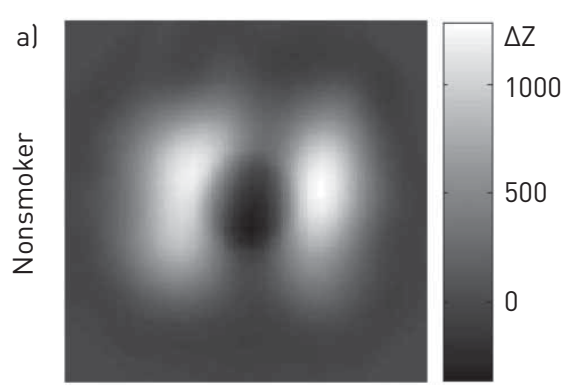

Female, 53 years, 0 pack-years

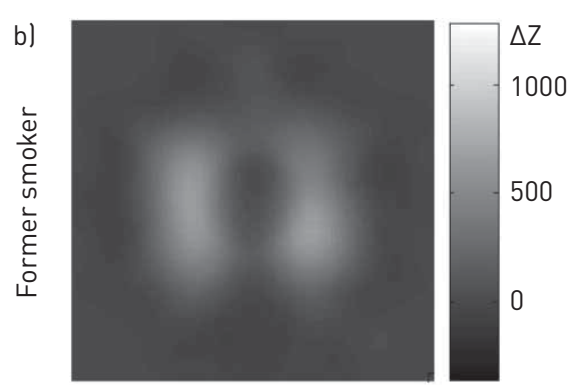

Female, 59 years, 21 pack-years smoking cessation for 12 years

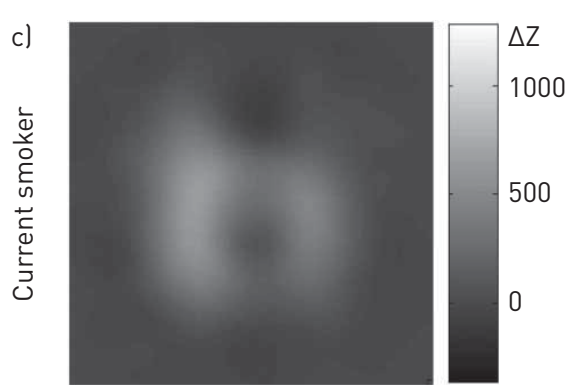

Male, 55 years, 45 pack-years

FIGURE 2 Functional electrical impedance tomography images presenting the regional ventilation distribution during forced expiration corresponding to the forced vital capacity manoeuvre determined in the chest cross section of three lung-healthy asymptomatic adults: a) nonsmoker, b) former smoker and c) current smoker. $\Delta \mathrm{Z}$ : relative impedance change. 
a)

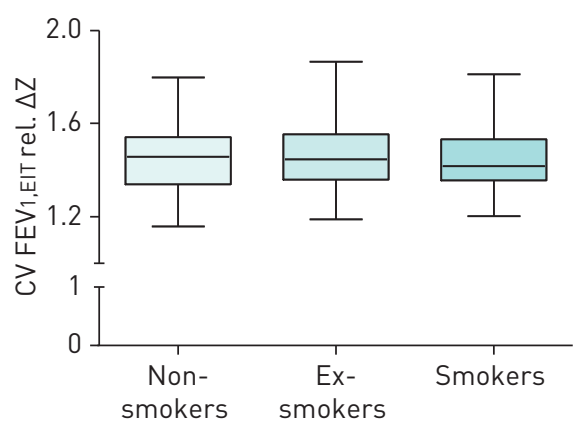

b)

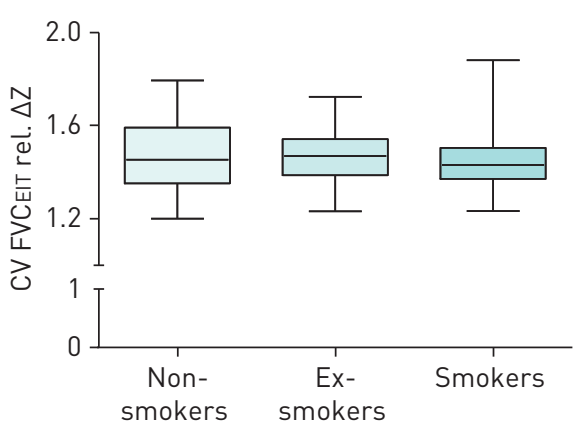

c)

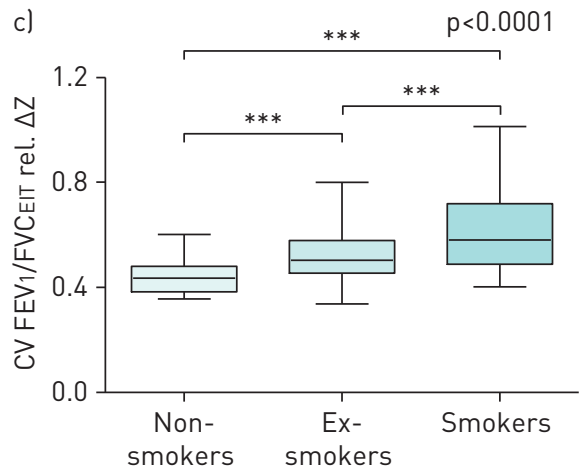

FIGURE 3 Coefficients of variation (CVs) of electrical impedance tomography (EIT)-derived regional pulmonary function measures of a) forced expiratory volume in $1 \mathrm{~s}(\mathrm{FEV} 1), \mathrm{b})$ forced vital capacity (FVC) and $\mathrm{c}$ ) FEV $1 / F V C$ determined in lung-healthy asymptomatic nonsmokers, and former and current smokers. The box and whiskers plots display the minimum, $25 \%$ percentile, median, $75 \%$ percentile and maximum values. The $\mathrm{p}$-value in the right upper corner of each diagram shows the result of statistical testing using a one-way ANOVA. Significant differences among the individual groups assessed by the Bonferroni's multiple comparison test are indicated by asterisks. rel. $\Delta Z$ : relative impedance change. ***: $p<0.0001$.
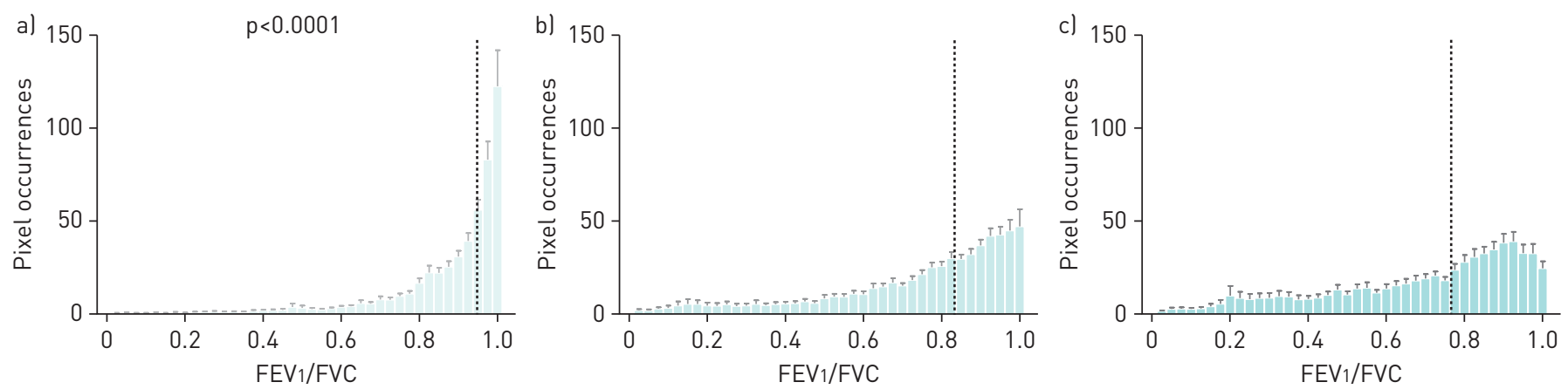

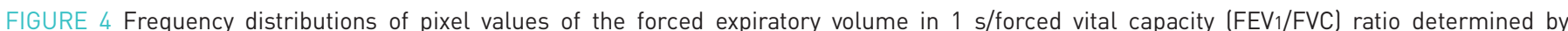
electrical impedance tomography in studied lung-healthy asymptomatic al nonsmokers, and b) former and c) current smokers. The histograms show mean+SEM. The vertical dotted lines correspond to the mean of the median values of the respective histograms. The $p$-value shows the result of statistical testing using one-way ANOVA. Significant differences among the studied groups assessed by the Bonferroni's multiple comparison test are indicated by asterisks. ${ }^{* * *}: \mathrm{p}<0.0001$. 
a)

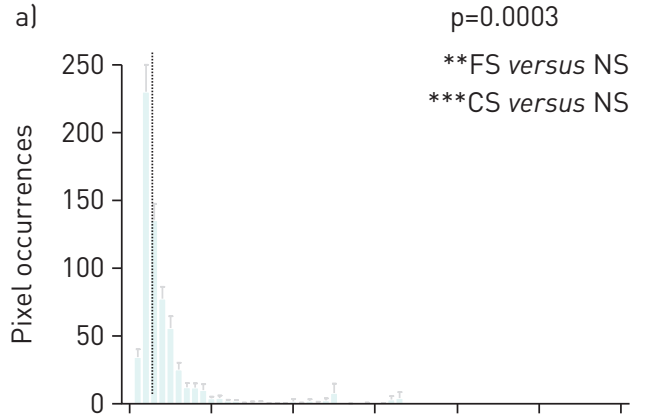

d)
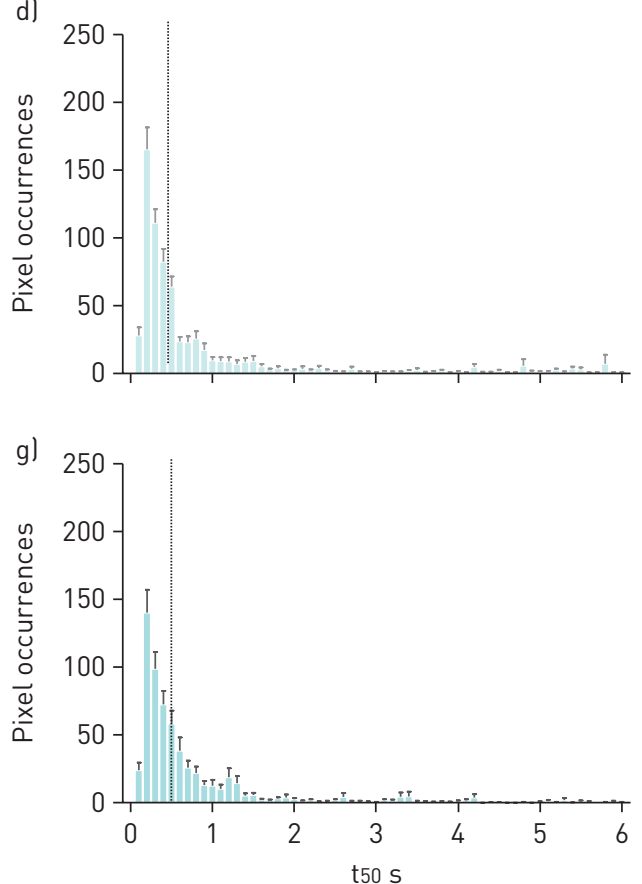

b)

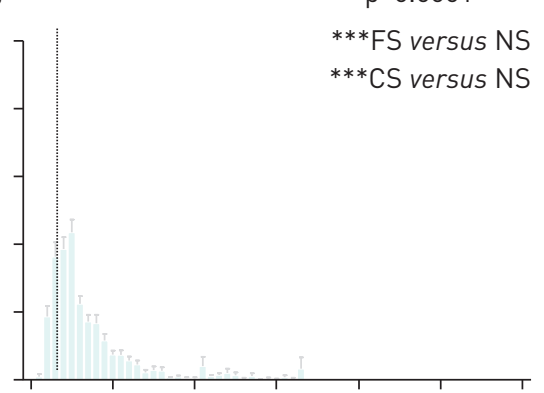

e)

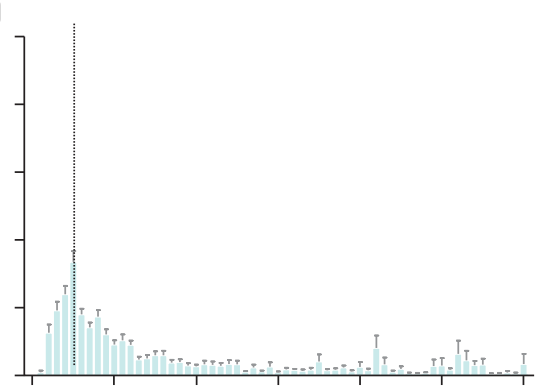

h)

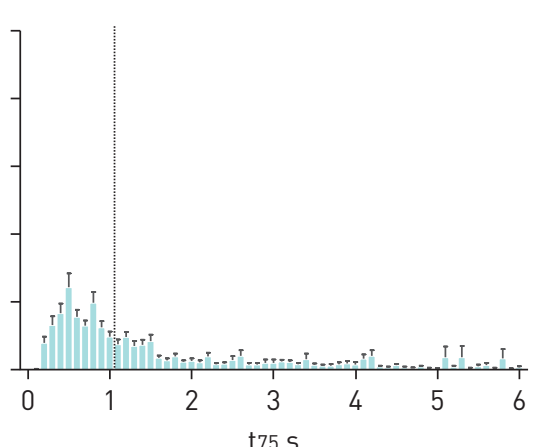

c)

$p<0.0001$

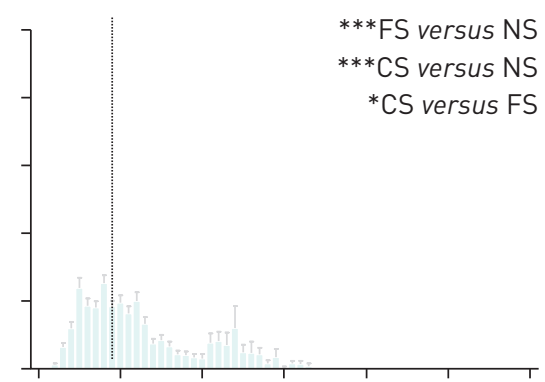

f)

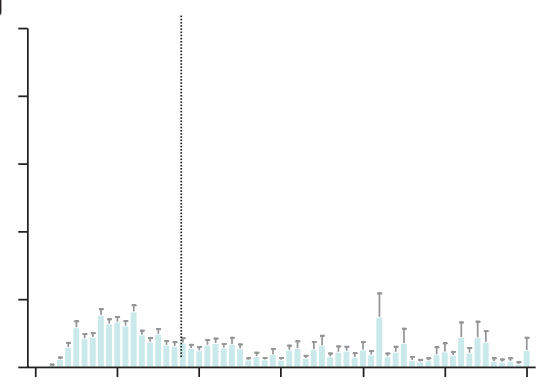

i)

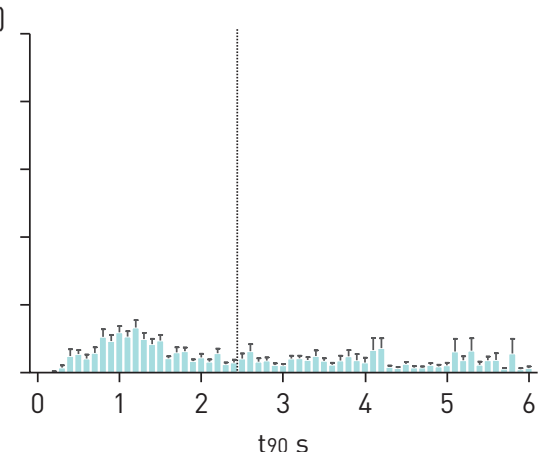

FIGURE 5 Frequency distributions of $a, d$ and g) pixel times required to exhale 50\% (t50) of regional forced vital capacities, b, e and h) pixel times required to exhale $75 \%$ (t75) of regional forced vital capacities and c, $f$ and i) pixel times required to exhale $90 \%$ (t90) of regional forced vital capacities determined by electrical impedance tomography in lung-healthy asymptomatic a-c) nonsmokers (NS), $d-f$ ) former smokers (FS) and $\mathrm{g}$-i) current smokers (CS). The histograms show mean values+SEM. The vertical grey dotted lines correspond to the mean of the median values of the respective histograms. The $p$-values of $t 50, t 75$ and 90 median in the corresponding columns show the results of statistical testing using one-way ANOVA. Significant differences among the groups assessed by the Bonferronís multiple comparison test are indicated by asterisks. *: $p<0.05 ;{ }^{* *}: p<0.001 ;{ }^{* *}: p<0.0001$.

The time-dependent functional information from PFT can be visualised by means of the regional lung function parameters $\mathrm{t50}, \mathrm{t} 75$ and $\mathrm{t} 90$. This allows not only the detection of differences in ventilation distribution among several study groups, as shown in our current study, but also timely changes in ventilation distribution between two measuring times in one individual such as before and after exercise challenge or before and after administration of bronchodilators as already shown in small studies [10-12]. In one of the first studies on PFT and EIT we detected differences in spatial and temporal ventilation distribution between lung-healthy subjects and patients with COPD [6]. The present study did not include patients with COPD; however, it still confirmed that EIT is sensitive enough to detect the even smaller regional differences in regional ventilation distribution in nonsmokers compared with asymptomatic past and current smokers. These study groups are not as clinically (concerning the pulmonary conditions) different from each other as the lung-healthy subjects and patients with COPD in the former study.

EIT is a functional imaging modality; it does not aim to provide anatomical information [5]. This is highlighted in figure 2 where the reduction in relative impedance change and the ventilation defects are only slightly visible. The spatial resolution of EIT is low when compared with other imaging modalities like magnetic resonance imaging (MRI) or CT. Sheikh et al. showed ventilation defects in never-smokers with normal spirometry results using hyperpolarised ${ }^{3} \mathrm{He}$ MRI [19]. Hamedani et al. used ${ }^{3} \mathrm{He}$ MRI to 
a)

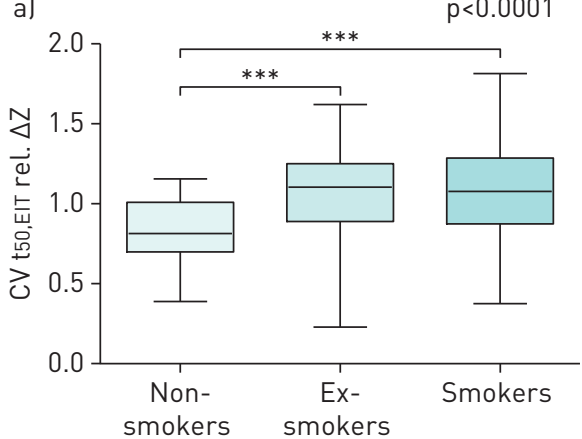

b)

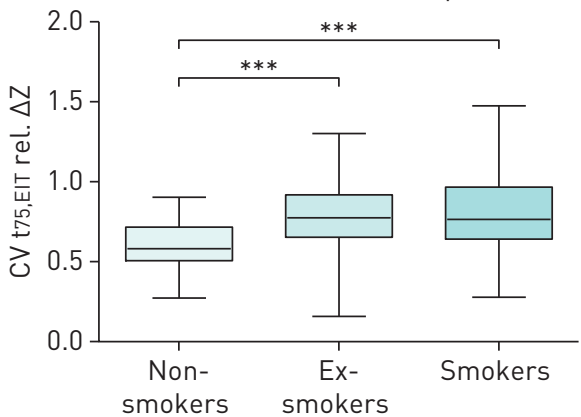

c)

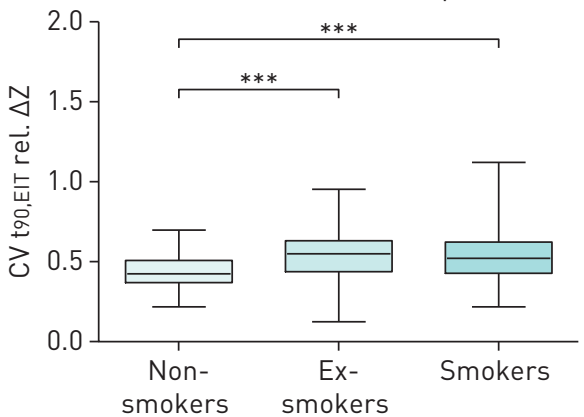

FIGURE 6 Coefficients of variation (CVs) of electrical impedance tomography (EIT)-derived regional a) pixel times required to exhale $50 \%$ (t50) of regional forced vital capacities, b) pixel times required to exhale $75 \%$ (t75) of regional forced vital capacities and c) pixel times required to exhale $90 \%$ (t90) of regional forced vital capacities determined in lung-healthy asymptomatic nonsmokers, former and current smokers. The box and whiskers plots display the minimum, $25 \%$ percentile, median, $75 \%$ percentile and maximum values. The p-value in the right upper corner of each diagram shows the result of statistical testing using a one-way ANOVA. Significant differences among the individual groups assessed by the Bonferroni's multiple comparison test are indicated by asterisks. rel. $\Delta Z$ : relative impedance change. ${ }^{* * *}: p<0.0001$.

prove that cigarette smoke induced pulmonary alterations that were not detectable by nonimaging clinical tests such as spirometry, plethysmography and the $6 \mathrm{~min}$ walk test (6MWT). They examined nonsmokers, asymptomatic smokers and patients with COPD and found no differences between nonsmokers and asymptomatic smokers when spirometry, plethysmography or 6MWT were applied [20]. Pike et al. were able to show very mild emphysema in ex-smokers without airway limitation by ${ }^{3} \mathrm{He}$ MRI [21]. New developments in CT allow differentiating among specific COPD phenotypes [22]. The anatomical information, such as dimensions of airways delivered by CT, correlates with the severity of airway obstruction [23]. In comparison with MRI and CT, EIT can be used at the bedside or in the PFT lab with lower costs regarding staff, equipment and examination time. Despite smaller scan rates than conventional PFT dominated by spirometry and plethysmography, EIT delivered data at a scan rate of 33 images per second in the present study, in up to 836 regional values of a certain regional lung function parameter, such as FEV1,EIT in the examined thorax cross section. Conventional PFT provides only one value, such as global FEV1 measured at the airway opening $[10,24]$.

As expected, in this study the proportion of women in the nonsmokers group was higher than in past and current smokers. Aside from the age of the past smokers and smoking history the study groups did not differ from each other. In the previous EIT study examining the ventilation heterogeneities in young and elderly nonsmokers, there were no differences found when CV was used [6]. We suspect that the age in the studied age range between 47 and 55 years had less influence on the regional lung function than the smoking history.

The nonsmokers group had a $20 \%$ higher proportion of women than men compared with the other study groups. We did not find any significant differences in the determined parameters of ventilation

TABLE 2 Values of electrical impedance tomography (EIT) variables in the three study groups.

\begin{tabular}{|c|c|c|c|c|}
\hline & Nonsmokers & Former smokers & Current smokers & p-value \\
\hline Median t5o & $0.27(0.21-0.33)$ & $0.33(0.27-0.48)^{\#}$ & $0.35(0.27-0.67)^{9}$ & 0.0003 \\
\hline Median $t_{75}$ & $0.30(0.25-0.38)$ & $0.48(0.37-0.77)^{\#}$ & $0.86(0.57-1.33)^{\pi}$ & $<0.0001$ \\
\hline Median t9o & $0.82(0.67-1.15)$ & $1.41(1.03-2.21)^{\#}$ & $1.91(1.33-3.53)^{9+}$ & $<0.0001$ \\
\hline Median FEV $1 /$ FVCEIT & $0.97(0.93-0.99)$ & $0.87(0.78-0.96)^{\#}$ & $0.84(0.69-0.92)^{9}$ & $<0.0001$ \\
\hline CV FEV1/FVCEIT & $0.43(0.38-0.48)$ & $0.47(1.38-1.54)^{\#}$ & $0.58(0.49-0.72)^{9++}$ & $<0.0001$ \\
\hline CV t50 & $0.81(0.70-1.01)$ & $1.10(0.89-1.25)^{\#}$ & $1.08(0.87-1.29)^{9}$ & $<0.0001$ \\
\hline CV t75 & $0.58(0.51-0.72)$ & $0.78(0.66-0.92)^{\#}$ & $0.77(0.64-0.97)^{\pi}$ & $<0.0001$ \\
\hline CV t9o & $0.42(0.37-0.50)$ & $0.54(0.43-0.63)^{\#}$ & $0.52(0.42-0.62)^{\pi}$ & $<0.0001$ \\
\hline
\end{tabular}

Data are presented as median (interquartile range) unless otherwise stated. t50, t75 and t90: times needed to expire $50 \%, 75 \%, 90 \%$ of forced vital capacity; FEV1: forced expiratory volume in $1 \mathrm{~s}$; FVC: forced vital capacity; CV: coefficient of variation. " : significant differences between former smokers and nonsmokers; ๆ: significant differences between current smokers and nonsmokers; ${ }^{+}$: significant differences between current and former smokers. 
heterogeneity between men and women in the three study groups. That is why we assume that the higher number of women than men was not the cause of more homogenous ventilation distribution in the nonsmoking study participants. However, studies on much higher number of subjects are needed to prove conclusively that sex does not affect the heterogeneity of ventilation distribution. In addition, other parameters may be needed to separately assess any possible effect of sex.

In order to adequately interpret changes in regional ventilation distribution in patients with pulmonary diseases, we need data from lung-healthy subjects and knowledge about its meaning. The basic research on heterogeneity of ventilation distribution during spontaneous breathing by EIT is new in the field of pneumology and must be systematically examined, as stated in the recent consensus statement on chest EIT [5]. The current study presents the first steps in this direction. The need for reference values in PFT has increased in recent years, as initially shown by Quanjer et al. for spirometry [25]. For years, reference values were used with a fixed FEV1/FVC ratio $<0.7$ for the detection of airway obstruction [26]. In 2012 the Global Lung Initiative introduced new spirometric reference values collected on 98000 healthy nonsmokers [25] that allowed more correct and personalised diagnostics of pulmonary function.

The present study was carried out in spontaneously breathing lung-healthy subjects, but the long-term goal is to establish EIT as a diagnostic and monitoring method in patients with chronic lung diseases. EIT has the potential for this use not only in the clinical hospital and outpatient settings but also in the preclinical home environment. Wearable EIT systems are being developed that might facilitate this application of EIT in the future [27]. Even with the currently available technology, the EIT examinations are not time-consuming. The attachment of electrodes is the most extensive work, whereby it takes less than 5 min for experienced personnel and this time can further be reduced if electrode belts are used. The duration of EIT data acquisition is based on the needs defined by the medical question. If EIT data are recorded only during the FVC manoeuvre then the EIT measurement takes just as long as when conventional spirometry is used, usually about $90 \mathrm{~s}$. During continuous patient examinations data collection over hours is possible.

Our study generated reference values of several regional EIT lung function parameters. The examinations were performed in parallel with conventional PFT (such as the FVC manoeuvre). This approach was chosen as it may facilitate the attempted establishment of EIT in the field of pneumology because the same well-known lung function parameters are determined by EIT as by spirometry, only on a regional level. However, additional new measures could be calculated ( $t 50, \mathrm{t} 75$ and $\mathrm{t} 90$ ) from EIT recordings in our study capable of characterising the heterogeneity of regional lung function. We suppose that further lung function measurements specifically designed for EIT use will be developed in the future. They should be easily carried out by patients and independent of their cooperation.

The number of our study participants is limited when compared with the mentioned large studies on conventional PFT. However, this study is the largest one ever carried out in adults for the determination of regional lung function by EIT worldwide to the best of our knowledge. The majority of the existing EIT studies on ventilation distribution focus on mechanically ventilated intensive care patients $[14,28-30]$. We believe that EIT chest examinations may be beneficial in spontaneously breathing patients with pulmonary diseases like COPD, $[6,31]$ asthma $[12,13,32]$ or cystic fibrosis $[11,33,34]$ as well and that this new functional method has a potential in pneumology.

The EIT impedance changes correlate strictly with spirometric data during the forced expiration manoeuvre [35]. Therefore, EIT can be used as a diagnostic tool providing the information about the regional pulmonary function in addition to the global one determined by conventional PFT. The new gained knowledge about the regional lung function might enable comprehensive individualised medicine.

Our study has some limitations that we wish to address. EIT measurements were carried out with the electrodes placed on the chest circumference in one plane. When EIT electrodes are located between the 4th and the 6th intercostal space, the apical lung regions and the areas close to diaphragm are not accessible to EIT examination. The optimal solution would be the simultaneous examination in multiple levels. To date, there are no devices available that would make this possible. Efforts exist to develop such technology [36] but the corresponding data acquisition and simultaneous processing at multiple levels are currently still difficult and not yet established.

We used single electrodes instead of EIT belts for data collection in our study. At first sight this might seem disadvantageous but, according to our experience, the EIT belts sometimes lose skin contact during the FVC manoeuvre due to the very quick decrease in the thoracic cross section. For each EIT measurement, the skin electrode contact and electrode location for current injection and detection of voltage changes should ideally not change with time. That is why we decided to use the single electrodes instead of EIT belts, although the belts are more frequently used in adults, especially in mechanically ventilated lying patients. 
Another limitation of this study is the lower proportion of smokers than past smokers and nonsmokers. Most smokers develop pulmonary complaints over time and they become symptomatic. We selected current smokers that were still asymptomatic and that did not yet receive the diagnosis of COPD. The recruitment at this stage is difficult because asymptomatic smokers have no reason to undergo medical consultation and treatment.

Our results support the hypothesis that only EIT-derived regional lung function parameters originating from lung-healthy nonsmokers should serve as reference values for regional ventilation distribution for patients with lung diseases. Smoking impacts the ventilation distribution patterns during forced expiration manoeuvre rendering them not compatible with values of homogeneous ventilation distribution observed in nonsmokers.

\section{Conclusions}

The results of our study highlight the potential of EIT as an additional tool in PFT for detecting regional ventilation distribution and its heterogeneity. In this study, we established the first EIT-derived regional lung function values for lung-healthy adults. Furthermore, we showed the impact of tobacco consumption on regional lung function. The regional EIT parameters might enable the discrimination between lung-healthy subjects and patients affected by lung diseases. The regional lung function results determined in patients should only be compared with results originating from nonsmoking lung-healthy reference population when EIT is used.

Conflict of interest: B. Vogt reports grants from the European Commission outside the submitted work. K. Deuß has nothing to disclose. V. Hennig has nothing to disclose. Z. Zhao reports a consulting fee from Dräger Medical AG outside the submitted work. I. Lautenschläger has nothing to disclose. N. Weiler has nothing to disclose. I. Frerichs report grants from the European Commission, and a speaking fee and reimbursement for travel costs from Dräger, outside the submitted work.

\section{References}

1 Downie SR, Salome CM, Verbanck S, et al. Ventilation heterogeneity is a major determinant of airway hyperresponsiveness in asthma, independent of airway inflammation. Thorax 2007; 62: 684-689.

2 Mauri T, Eronia N, Abbruzzese C, et al. Effects of sigh on regional lung strain and ventilation heterogeneity in acute respiratory failure patients undergoing assisted mechanical ventilation. Crit Care Med 2015; 43: 1823-1831.

3 Sinclair SE, Polissar NL, Altemeier WA. Spatial distribution of sequential ventilation during mechanical ventilation of the uninjured lung: an argument for cyclical airway collapse and expansion. BMC Pulm Med 2010; $10: 25$.

4 Teague WG, Tustison NJ, Altes TA. Ventilation heterogeneity in asthma. J Asthma 2014; 51: 677-684.

5 Frerichs I, Amato MB, van Kaam AH, et al. Chest electrical impedance tomography examination, data analysis, terminology, clinical use and recommendations: consensus statement of the TRanslational EIT developmeNt stuDy group. Thorax 2017; 72: 83-93.

6 Vogt B, Pulletz S, Elke G, et al. Spatial and temporal heterogeneity of regional lung ventilation determined by electrical impedance tomography during pulmonary function testing. J Appl Physiol 2012; 113: 1154-1161.

7 Schullcke B, Krueger-Ziolek S, Gong B, et al. Ventilation inhomogeneity in obstructive lung diseases measured by electrical impedance tomography: a simulation study. J Clin Monit Comput 2018; 32: 753-761.

8 Vogt B, Mendes L, Chouvarda I, et al. Influence of torso and arm positions on chest examinations by electrical impedance tomography. Physiol Meas 2016; 37: 904-921.

9 Frerichs I, Braun P, Dudykevych T, et al. Distribution of ventilation in young and elderly adults determined by electrical impedance tomography. Respir Physiol Neurobiol 2004; 143: 63-75.

10 Vogt B, Lohr S, Zhao Z, et al. Regional lung function testing in children using electrical impedance tomography. Pediatr Pulmonol 2018; 53: 293-301.

11 Zhao Z, Fischer R, Frerichs I, et al. Regional ventilation in cystic fibrosis measured by electrical impedance tomography. J Cyst Fibros 2012; 11: 412-418.

12 Frerichs I, Zhao Z, Becher T, et al. Regional lung function determined by electrical impedance tomography during bronchodilator reversibility testing in patients with asthma. Physiol Meas 2016; 37: 698-712.

13 Ngo C, Dippel F, Tenbrock K, et al. Flow-volume loops measured with electrical impedance tomography in pediatric patients with asthma. Pediatr Pulmonol 2018; 53: 636-644.

14 Van der Burg PS, Miedema M, de Jongh FH, et al. Cross-sectional changes in lung volume measured by electrical impedance tomography are representative for the whole lung in ventilated preterm infants. Crit Care Med 2014; 42: 1524-1530.

15 Lehmann S, Leonhardt S, Ngo C, et al. Global and regional lung function in cystic fibrosis measured by electrical impedance tomography. Pediatr Pulmonol 2016; 51: 1191-1199.

16 Miller MR, Hankinson J, Brusasco V, et al. Standardisation of spirometry. Eur Respir J 2005; 26: 319-338.

17 Vogt B, Hennig V, Deuss K, et al. Performance of new spirometry reference values in preoperative assessment of lung function. Clin Respir J 2019; 13: 239-246.

18 Adler A, Arnold JH, Bayford R, et al. GREIT: a unified approach to 2D linear EIT reconstruction of lung images. Physiol Meas 2009; 30: S35-S55.

19 Sheikh K, Paulin GA, Svenningsen S, et al. Pulmonary ventilation defects in older never-smokers. J Appl Physiol 2014; 117: 297-306.

20 Hamedani H, Shaghaghi H, Kadlecek SJ, et al. Vertical gradients in regional alveolar oxygen tension in supine human lung imaged by hyperpolarized 3He MRI. NMR Biomed 2014; 27: 1439-1450. 

2015; 22: 1068-1078.

22 Galban CJ, Han MK, Boes JL et al. Computed tomography-based biomarker provides unique signature for diagnosis of COPD phenotypes and disease progression. Nat Med 2012; 18: 1711-1715.

23 Lynch DA, Al-Qaisi MA. Quantitative computed tomography in chronic obstructive pulmonary disease. $J$ Thorac Imaging 2013; 28: 284-290.

24 Vogt B, Falkenberg C, Weiler N, et al. Pulmonary function testing in children and infants. Physiol Meas 2014; 35: R59-R90.

25 Quanjer PH, Stanojevic S, Cole TJ, et al. Multi-ethnic reference values for spirometry for the 3-95-yr age range: the global lung function 2012 equations. Eur Respir J 2012; 40: 1324-1343.

26 Vogelmeier CF, Criner GJ, Martinez FJ, et al. Global Strategy for the Diagnosis, Management and Prevention of Chronic Obstructive Lung Disease 2017 report: GOLD executive summary. Respirology 2017; 22: 575-601.

27 Rapin M, Braun F, Adler A, et al. Wearable sensors for frequency-multiplexed EIT and multilead ECG data acquisition. IEEE Trans Biomed Eng 2019; 66: 810-820.

28 Becher T, Kott M, Schadler D, et al. Influence of tidal volume on ventilation inhomogeneity assessed by electrical impedance tomography during controlled mechanical ventilation. Physiol Meas 2015; 36: 1137-1146.

29 Victorino JA, Borges JB, Okamoto VN, et al. Imbalances in regional lung ventilation: a validation study on electrical impedance tomography. Am J Respir Crit Care Med 2004; 169: 791-800.

30 Pulletz S, Adler A, Kott M, et al. Regional lung opening and closing pressures in patients with acute lung injury. J Crit Care 2012; 27: 323 e11-8.

31 Vogt B, Zhao Z, Zabel P, et al. Regional lung response to bronchodilator reversibility testing determined by electrical impedance tomography in chronic obstructive pulmonary disease. Am J Physiol Lung Cell Mol Physiol 2016; 311: L8-L19.

32 Schullcke B, Krueger-Ziolek S, Gong B, et al. Compensation for large thorax excursions in EIT imaging. Physiol Meas 2016; 37: 1605-1623.

33 Zhao Z, Muller-Lisse U, Frerichs I, et al. Regional airway obstruction in cystic fibrosis determined by electrical impedance tomography in comparison with high resolution CT. Physiol Meas 2013; 34: N107-N114.

34 Lehmann S, Tenbrock K, Schrading S, et al. Monitoring of lobectomy in cystic fibrosis with electrical impedance tomography - a new diagnostic tool. Biomed Tech 2014; 59: 545-548.

35 Ngo C, Leonhardt S, Zhang T, et al. Linearity of electrical impedance tomography during maximum effort breathing and forced expiration maneuvers. Physiol Meas 2017; 38: 77-86.

36 Wagenaar J, Adler A. Electrical impedance tomography in 3D using two electrode planes: characterization and evaluation. Physiol Meas 2016; 37: 922-937. 